when it ruled that greenhouse gases from vehicles could be regulated under the Clean Air Act if the EPA determined that they posed a threat to humans - as the agency now proposes to do. It could start by setting a limit on greenhouse-gas emissions for all transportation fuels, or by setting carbon-based standards for energy-intensive industries.

As worthwhile as such efforts would be, however, nobody really wants the EPA to go solo on climate. Successfully reducing US carbon emissions by any amount, let alone meeting the administration's goal of cutting them to $80 \%$ of 1990 levels by mid-century, will require a framework of law, regulations and incentives that encompass all of US society. And for action of that magnitude, the EPA and every other relevant agency should have the imprimatur of society's representatives in Congress.

In an alternative scenario - the one that advocates of climate legislation are hoping for - the looming threat of the EPA's regulatory authority would nudge all sides to the table for a grand congressional bargain on greenhouse-gas regulations. The presumption is that business leaders, Republicans, coal-state Democrats and others wary of climate regulation will figure that they have a better chance of protecting their interests in Congress than in the deliberations of EPA technocrats. Supporters, meanwhile, will figure that a congressional stamp of approval will help to avoid long legal challenges about every detail of the regulations, as well as the prospect of a loss in court. (Just such an outcome occurred last year when a fight over regulatory details led a federal appeals court to toss out an emissions-trading programme designed to lessen the amount of smog that drifts east from power plants in the Midwest.)

In the meantime, President Barack Obama has rightly chosen to focus his climate-change efforts on Capitol Hill, where several bills have already been introduced to regulate greenhouse gases by a capand-trade system. Unfortunately, his administration hasn't exactly been crystal clear about what it expects from Congress and when. This is perhaps understandable, given the energy that Obama has been forced to dedicate to the ongoing economic crisis, but the administration will eventually have to wade into the debate, get involved in the negotiations and demonstrate real leadership in this area.

The good news is that Obama seems to understand the complexities of the problem. His science adviser John Holdren recently told Nature that Obama has shown "a willingness and an ability to keep issues together when they need to be together". Irrespective of how popular his policies might be, there is little doubt that Obama has shown a bold - some would say brash — inclination to break with the stove-piped policymaking of the past. Energy and climate, for example, had for some time been handled separately but are now part of a single discussion that includes competitiveness and economic recovery. Still, comprehensive reform will always be more difficult than a piecemeal approach. In his short time in office, Obama has showed his willingness to push for reform on numerous fronts. Now he needs to prove he can follow through.

\title{
No more catch-up
}

\section{Regulatory agencies need to be more proactive in preparing for avant-garde products.}

$\uparrow$ he London-based European Medicines Agency (EMEA), the body in charge of regulating the marketing of pharmaceuticals in Europe, is falling well short of its stated goal of stimulating the development of innovative new medicines.

In particular, the EMEA has stumbled in its dealings with PharmaPlanta, an academia-led consortium funded by the European Commission (EC) to develop drugs for diseases that continue to pose problems in both Europe and the developing world using transgenic plants.

The consortium recently offered to help the EMEA improve regulations for the manufacture of plant-made drugs, so that they would be ready when the first products come to market (see page 951). It also hoped to instil confidence in the wider European drug industry, which has been reluctant to invest in drugs made from transgenic plants despite the technique's promise as an inexpensive drug-production pathway. One reason for this reluctance may be that many Europeans are reluctant to embrace genetically modified crops in general. But a large part of the problem, analysts say, is that the strict controls on manufacturing processes and product quality required to get marketing permission from the EMEA were drawn up for traditional biopharmaceutical production methods, which use engineered microbes and cultured mammalian cells. Those controls needed to be redrafted to fit transgenic plant systems.
Hence the consortium's offer. The EMEA, however, insisted on charging Pharma-Planta its standard rate for scientific advice $€ 75,500$ (US\$97,500 ) - because it had no policy in place to give academic institutions the same $90 \%$ discount that it gives small businesses. The EMEA did offer a 50\% discount, but even so the consortium had to bow out; it could not afford to work with the agency.

The EMEA should reconsider this position. It is one thing to charge a premium fee to large pharmaceutical companies looking to market a new drug with a huge profit potential. But publicly funded institutions can no more afford those premium fees than small businesses can. Moreover, the Pharma-Planta consortium wasn't trying to market anything; it simply wanted to help the EMEA to develop better regulation and guidelines. The EMEA has now published

\section{"Publicly funded institutions can no more afford premium fees than small businesses can."}

improved guidelines, albeit with limited input from Pharma-Planta, the leading expert in the field.

The EMEA should also reconsider the process through which it develops its guidelines and regulation. It holds an important and influential position in Europe: the overarching guidelines and regulations it produces filter down and often inform national rules across the continent. The EMEA must be more proactive in developing guidance on up-and-coming technologies - especially on new and controversial ones such as transgenic-plant-derived pharmaceuticals - rather than waiting for others to point out that new rules are needed. 